\title{
EFFECT OF NON-THERMAL AIR PLASMA TREATMENT ON SHEAR BOND STRENGTH OF ADHESIVE RESIN CEMENT TO ZIRCONIA
}

\author{
Aliaa Mahrous*, Mohamed M Radwan ${ }^{* *}$ and Bassem Emad $^{* * * *}$
}

\begin{abstract}
Purpose: The purpose of the present study was to analyze the effect of non-thermal air plasma treatment on shear bond strength and failure mode of self-adhesive resin cement to zirconia.

Materials\& Methods: Forty eight zirconia plates were prepared from IPS e.max ZirCad blocks (Ivoclar Vivadent, Schaan, Liechtenstein) and classified into four groups $(n=12)$ according to surface treatment; group CO (control) without any surface treatment, group SD was treated with $50 \mu \mathrm{m}$ alumina sandblasting, group PL used air atmospheric pressure plasma device (Piezobrush ${ }^{\circledR}$ PZ2, Relyon plasma GMBH, Regensburg, Germany) \& group SP which was a combination of $50 \mu \mathrm{m}$ alumina sandblasting followed immediately with atmospheric plasma treatment. Zirconia specimens were immediately centered with Relay X Unicem (3M ESPE) resin cylinders of $3.7 \mathrm{~mm}$ diameter and $2 \mathrm{~mm}$ height. Shear bond strength test was performed 8 days after water storage at $37^{\circ} \mathrm{C}$ using a computer controlled materials testing machine (Model 3345; Instron Industrial Products, Norwood, USA). A scanning electron microscope (Quanta 250 Field Emission Gun, Netherlands) was used to determine failure mode with all morphological and structural changes. Average surface roughness $(\mathrm{Ra})$ was calculated with different surface treatments. Energy Dispersive X-ray (EDX) analysis was used for chemical changes evaluation. Data were analyzed with one-way ANOVA followed by Tukey's post hoc test when the ANOVA test was significant.
\end{abstract}

Results: A significant difference between different surface treatments $(\mathrm{P}=0.010)$ was recorded with the highest shear bond strength value in SP group and the lowest value with untreated surface CO gp. All groups showed mixed failure mode which was mainly cohesive except for CO gp. Surface roughness was increased with sandblasting and decreased after plazma treatment. EDX analysis showed increase oxygen on plazma treated surface.

Conclusion: Non thermal air plasma treatment can be used for increasing zirconia surface treatment especially when accompanied with $50 \mu \mathrm{m} \mathrm{AL}_{2} \mathrm{O}_{3}$ sandblasting.

Clinical significant: Non thermal air plasma treatment alone or in combination with sandblasting can be considered as a good clinical protocol for cementing zirconia restorations.

Keywords Atmospheric air plasma treatment, sandblasting, Relay X Unicem, zirconia, shear bond strength test (SBST), failure mode, surface roughness.

* Lecturer Fixed Prosthodontics Department, Faculty of Dentistry, Fayoum University, Egypt.

** Lecturer Fixed Prosthodontics Department, Faculty of Dentistry, Beni Suef University, Egypt.

*** General Oral and Maxillofacial Surgery Practioner, Private Institution,Egypt. 


\section{INTRODUCTION}

Widespread application of zirconia based restorations in prosthetic dentistry with its unique mechanical properties, excellent biocompatibility and esthetics have been greatly increased('). The high strength of zirconia restorations can render the material as a favorable selection especially for minimal invasive restorations with required adhesive procedures ${ }^{(2)}$. The clinical success here is strongly dependent on adhesive bond strength and its long term stability ${ }^{(3)}$.

It is a continuous challenge to produce a proper bonding of zirconia and other substrates because of its chemical stability with hydrophobic nature ${ }^{(4 \& 5)}$. Moreover, the increase in crystalline content of zirconia reducing the glass phase with possible etching effect. The problem is mostly determined with less retentive restoration of $100 \mathrm{um} \mathrm{misfit}^{(6)}$.

Many approaches have been tried for enhancing the adhesive resin bond with high crystalline zirconia restorations $^{(3)}$. Most of these approaches have worked on increasing roughness of zirconia surface either by tribochemical silica coating followed by the application of the trialkoxysilane coupling agents ${ }^{(7)}$ or using airborne $\mathrm{Al}_{2} \mathrm{O}_{3}$ particle abrasion followed by the application of phosphate ester monomer-containing primers such as 10-methacryloyloxydecyl dihydrogen phosphate $(\mathrm{MDP})^{(8)}$.

Unfortunately, some studies recorded creation of critical flaws in the zirconia ceramic surface with many approaches resulting in catastrophic failure and this explained the clinical failures of some documented cases ${ }^{(9 \& 10)}$.

In recent years, plasma applications in dentistry as a novel surface treatment have attracted much attention. Plasma is defined as the $4^{\text {th }}$ state of matter formed of atoms, molecules and highly excited radicals. Plasma has different forms including natural and artificial types. It also can be classified according to their parameters. At atmospheric pressure level, there is a thermal type and cold plasma (non-thermal) with gas temperature close to room temperature ${ }^{(11)}$.

The cold plasma treatment is a technique used for chemical surface modification and carried out on molecular level without affecting bulk performance of the material ${ }^{(12 \& 13)}$.

Furthermore, plasma can induce surface energy and remove all contaminations leading to improvement in surface wettability ${ }^{(14)}$. The technique formed of partially ionized gas in non-equilibrium providing chemically active species in large amount and under low temperature. These species are such as $\mathrm{O} 3, \mathrm{OH}, \mathrm{H} 2 \mathrm{O} 2, \mathrm{NO}, \mathrm{OH}$ radicals which can change non-reactive functional group of the surface to reactive radicals that enhance bonding ${ }^{(15-17)}$.

It has been suggested to apply cold atmospheric plasma as a promising technique in dental field for adhesive bonding improvement with zirconia restorations ${ }^{(5)}$.

The objective of the present study was to analyze the effect of non-thermal atmospheric air plasma treatment on SBS and failure mode of self-adhesive resin cement to zirconia. Moreover, examining the surface properties with morphological and chemical change.s The null hyposthesis was that with plasma treatment the shear bond strength of zirconia will be enhanced.

\section{MATERIALS \& METHODS}

\section{Experimental design}

Forty eight zirconia plates were prepared from IPS e.max ZirCad blocks (Ivoclar Vivadent, Schaan, Liechtenstein) using diamond saw (IsoMet 4000 linear Precision saw, Buehler, USA) under copious water coolant. Zirconia specimens were sintered in an InFire HTC speed high-temperature furnace (Sirona Dental System GmbH, Germany). 
The zirconia specimens were embedded in split cylindrical tefflon molds filled with self-cure acrylic resin with the treated surface facing upward for testing. The tested zirconia surfaces were polished with 600 to 1200 grit silicon carbide paper (Sailbrand, China) under continuous use of water coolant. Finally before surface treatment, all the specimens were ultrasonically cleaned in distilled water for 5 minutes before bonding procedures.

The zirconia specimens were classified into four groups ( $n=12)$ according to surface treatment; group $\mathrm{CO}$ (control) without any surface treatment, group $\mathrm{SD}\left(\mathrm{AL}_{2} \mathrm{O}_{3}\right.$ sandblasting surface treatment), group PL (air atmospheric plasma treatment) \& group $\mathrm{SP}\left(\mathrm{AL}_{2} \mathrm{O}_{3}\right.$ sandblasting + air atmospheric plasma treatment).

\section{Surface treatment}

1- Control group (CO): zirconia specimens were kept for resin cementation and testing without any surface treatment.

2- SD group: zirconia specimens were sandblasted (Renfert Basic Blassic, Renfert GmbH, Germany) with $50 \mu \mathrm{m} \mathrm{AL}_{2} \mathrm{O}_{3}$ particles from a $10 \mathrm{~mm}$ distance for 10 seconds under $0.3 \mathrm{MPa}$ pressure.

3- PL group: specimens were treated with nonthermal air atmospheric plasma using atmospheric pressure plasma device (Piezobrush ${ }^{\circledR}$ PZ2, Relyon plasma GMBH, Regensburg, Germany) with about $5 \mathrm{~mm}$ distance from the nozzle tip to the treated surface for a total of 80 seconds at a maximum power consumption of $30 \mathrm{~W}$ and a temperature of $\sim 50^{\circ} \mathrm{C}$.

4- SP group: specimens were treated by using $50 \mu \mathrm{m}$ alumina sandblasting for 10 seconds under 0.3 MPa pressure from a $10 \mathrm{~mm}$ distance followed immediately by atmospheric plasma treatment for 80 seconds with $5 \mathrm{~mm}$ distance at a maximum power consumption of $30 \mathrm{~W}$ and a temperature of $\sim 50^{\circ} \mathrm{C}$.

\section{Bonding procedures}

Nine specimens of each group $(n=9)$ were immediately centered with RelayX Unicem (3M ESPE) resin cylinder guided by translucent polyethylene mold with $3.7 \mathrm{~mm}$ inner diameter and $2 \mathrm{~mm}$ height. Resin cement was mixed according to manufacturer instructions, packed and light cured for 20 seconds from each side (Lunar Curing Light, Benlioglu Dental Inc., Ankara, Turkey). Residual resin cement was cleaned by a microbrush before light polymerization. After removal of the mold, all resin cement cylinders were checked for bonding interface defects. Zirconia specimens were water stored for 8 days at $37^{\circ} \mathrm{C}$ before mechanical testing.

\section{Shear bond strength test (SBST)}

A circular interface shear test was designed to evaluate the bond strength. Each zirconia specimen was horizontally mounted on a computer controlled materials testing machine (Model 3345; Instron Industrial Products, Norwood, USA) with a loadcell of $5 \mathrm{kN}$ and data was recorded using computer software (Bluehill Lite; Instron Instruments). The specimen was secured to the lower fixed compartment of testing machine by tightening screws. Shearing test was done by compressive mode of load applied at zirconia-resin interface using a mono-angled chisel shaped metallic rod attached to the upper movable compartment of testing machine traveling at cross-head speed of 0.5 $\mathrm{mm} / \mathrm{min}$. The load required for dislodging the resin cylinder was recorded automatically in Newton. The shear bond strength was calculated according to the following equation; $\boldsymbol{\tau}=\mathbf{P} / \pi \mathbf{r}^{2}$

Where; $\tau$ was the shear bond strength in MPa, $\mathrm{P}$ was the load at failure in $\mathrm{N}, \pi$ was a constant equal to 3.14 and $\mathrm{r}$ was the radius of disc $(\mathrm{mm})$.

\section{Scanning Electron Microscope}

Zirconia specimens of all group were examined for failure mode determination using Scanning 
Electron Microscope (Quanta 250 Field Emission Gun, Netherlands) at $60 \mathrm{X}$ magnification. Represented areas of every failure mode were inspected with higher magnifications (X 500 ) to record morphological and ultra-structural changes.

\section{Surface roughness \&EDX analysis}

Three specimens of each group $(n=3)$ were used for Topographic evaluation immediately after surface treatment. Roughness parameters were determined using Scandium software (Scandium Olympus soft image solutions $\mathrm{GmbH}$, USA) at three different areas of each of specimen. Energy Dispersive X-ray (EDX) unit attached with SEM was used to analyze the chemical changes after each surface treatment with accelerating voltage of $30 \mathrm{~K} . \mathrm{V}$., magnification $14 \mathrm{x}$ up to 1000000 and resolution for Gun.1n with a secondary electron detector.

\section{Statistical analysis:}

Numerical data were explored for normality by checking the data distribution, calculating the mean and median values and using Kolmogorov-Smirnov and Shapiro-Wilk tests. Data showed parametric distribution so; it was represented by mean and standard deviation (SD) values. One-way ANOVA was used for intergroup comparisons followed by Tukey's post hoc test when the ANOVA test was significant. The significance level was set at $\mathrm{P} \leq 0.05$ for all tests. Statistical analysis was performed with with IBM ${ }^{\circledR}$ SPSS ${ }^{\circledR}$ (SPSS Inc., IBM Corporation, NY, USA) Statistics Version 25 for Windows.

\section{RESULTS}

\section{Shear bond strength}

A significant difference between different surface treatments $(\mathrm{P}=0.010)$ was recorded (Figure 1). SP group (sandblasting + plasma treatment) had the highest $($ mean \pm SD) value $(23.88 \pm 2.05)$ followed by PL group (plasma) (20.22 \pm 1.76$)$ and SD group (sandblasting) (15.09 \pm 1.55$)$. The untreated surface (control) had the least (mean \pm SD) value (11.23 \pm 1.75$)$. Pairwise comparison showed the SP group to be significantly different than $\mathrm{CO}$ group (control).

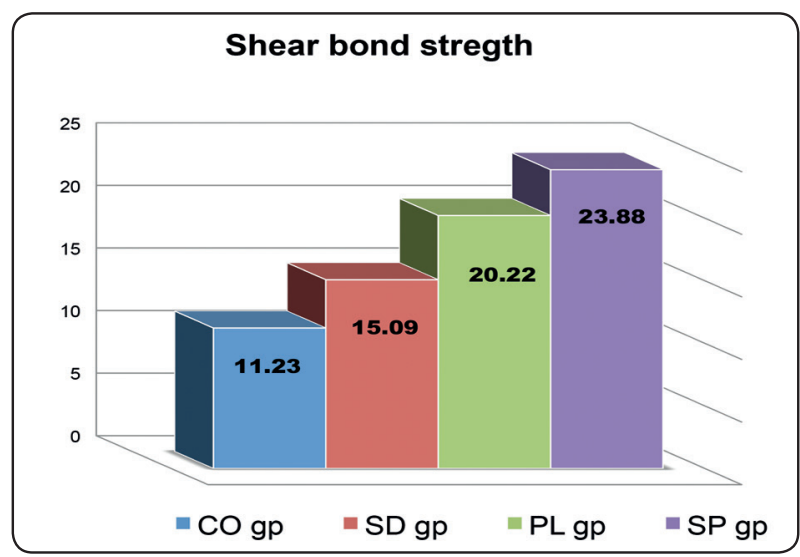

Fig. (1): Bar chart showing average shear bond strength (MPa) between different surface treatments.

\section{Scanning Electron Microscope}

All debonded specimens from groups (SP, PL, SD) showed mixed failure mode which was mainly cohesive (Figure 2B) except for the control group (CO) as the failure was mainly adhesive on the majority of the surface (Figure 2A).

Higher magnifications (X 500) of the debonded surfaces (Figure 3 ) showed a very limited area of resin in CO group (Figure 3A), widespread of resin in SD group (Figure 3B) with increased areas of adhered resin to the surface in PL \& SP group (Figure $3 C \& D)$. The resin in PL group was seemed to cover most of the surface and the uncovered area showed multiple small scattered resin islands while in SP group, resin looked as it was infiltrated in the whole surface covering the majority of it.

\section{Surface Roughness and EDX analysis}

There were a significant difference between different surface treatments $(\mathrm{P}<0.001)$. Group SP 


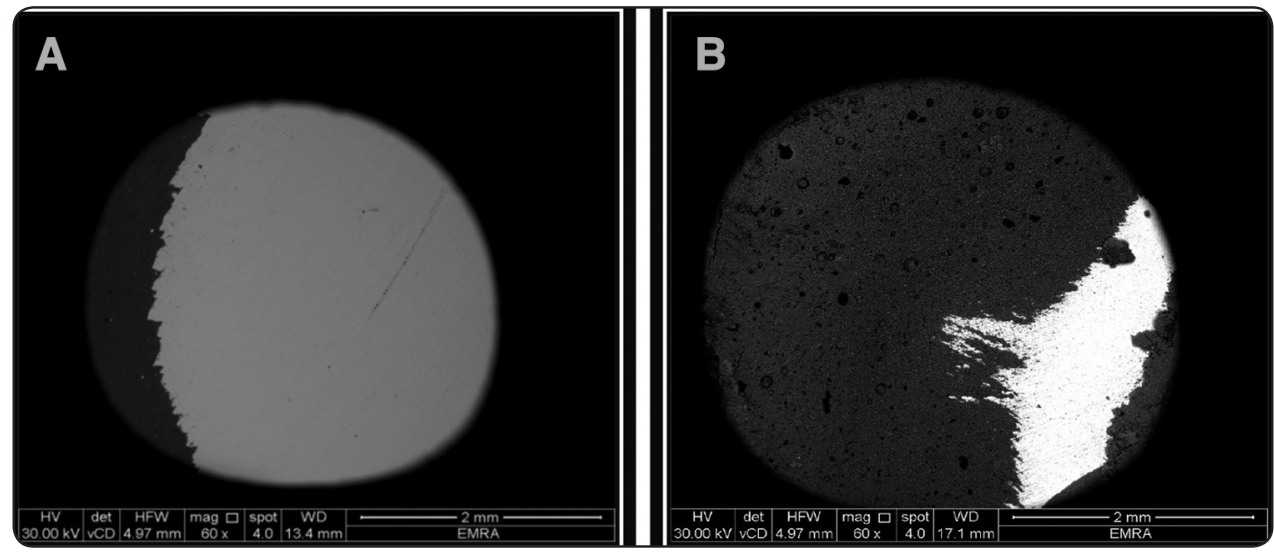

Fig. (2): Representative SEM (X60 magnification) showed mixed failure mode of the debonded surface in CO group (Figure 2A) Which was mainly adhesive \& in SP group (Figure 2B) which was cohesive mainly. Note that group SP (highest bond strength) has a very small areas of adhesive failure.
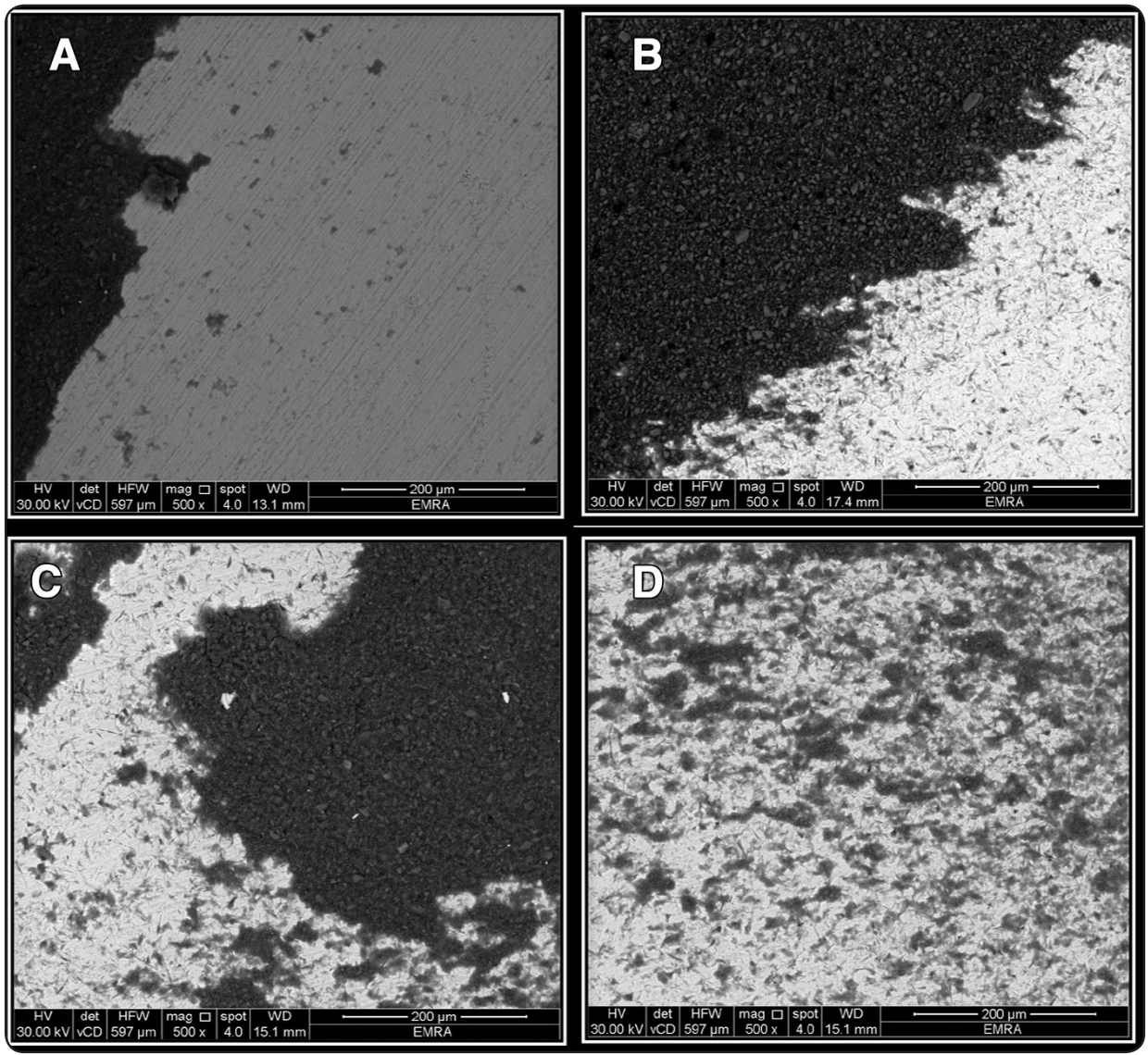

Fig. (3): Representative SEM (X500 magnification) morphologic characterization of debonded zirconia surface with very small area of remaining attached resin in the untreated surface $\mathrm{CO}$ gp (figure 3A), more spread of resin on sandblasted surface SD gp covering more than $50 \%$ of the surface (figure $3 \mathrm{~B}$ ), the resin with plasma treated surface PL gp extended on more areas with increased resin islands on the uncovered area (figure 3C) while the Sp gp showed resin to be infiltrated in the surface with scattered islands covering almost the whole surface (figure 3D). 
was $(19.43 \pm 0.43)$ with the highest $($ mean \pm SD) value (Figure4) followed by SD gp (18.61 \pm 0.49$)$ then and PL gp (14.76 \pm 0.30$)$. CO gp(control) was $(13.53 \pm 0.35)$ with the lowest $($ mean $\pm S D)$ value. Pairwise comparisons showed samples treated with different surface treatments to be significantly different $(\mathrm{P}<0.05)$ from each other except for group SD \& PL. (Table 1, Figure 4,5\&6)

EDX analysis showed increase in oxygen \% in PL group and SP while SD gp showed increase in oxygen and alumina \% (Table 2).

TABLE (1): Mean \pm SD of Surface roughness $(\mathrm{Ra})(\mu \mathrm{m})$ for different surface treatments

\begin{tabular}{|c|c|c|c|c|}
\hline \multicolumn{4}{|c|}{ Surface roughness (mean \pm SD) } & \multirow{2}{*}{ P-value } \\
\hline CO & SD & PL & SP & $<0.001^{*}$ \\
\hline $13.53 \pm 0.35^{\mathrm{A}}$ & $18.61 \pm 0.49^{\mathrm{B}}$ & $14.76 \pm 0.30^{\mathrm{C}}$ & $19.43 \pm 0.43^{\mathrm{B}}$ & $<$ \\
\hline
\end{tabular}

Different superscript letters within the same row indicates a statistically significant difference*; significant (p $\leq 0.05)$ ns; non-significant $(p>0.05)$

TABLE (2): Energy dispersive x-ray (EDX) of the different zirconia surface treatment

\begin{tabular}{|c|c|c|c|c|c|c|}
\hline \multirow[b]{2}{*}{ Element } & \multicolumn{2}{|c|}{ SD group } & \multicolumn{2}{|c|}{ PL group } & \multicolumn{2}{|c|}{ SP group } \\
\hline & $\mathrm{Wt} \%$ & At $\%$ & $\mathrm{Wt} \%$ & At $\%$ & $\mathrm{Wt} \%$ & At $\%$ \\
\hline $\mathrm{C} \mathrm{K}$ & 12.16 & 29.90 & 13.75 & 33.07 & 10.87 & 27.39 \\
\hline $\mathrm{OK}$ & 25.82 & 47.66 & 26.34 & 47.57 & 25.49 & 49.08 \\
\hline $\mathrm{AlK}$ & 3.05 & 3.34 & 0.50 & 0.45 & 3.25 & 3.64 \\
\hline $\mathrm{Zr} \mathrm{K}$ & 58.97 & 19.09 & 59.41 & 18.82 & 59.94 & 19,89 \\
\hline Total & 100.00 & 100.00 & 100.00 & 100.00 & 100.00 & 100.00 \\
\hline
\end{tabular}

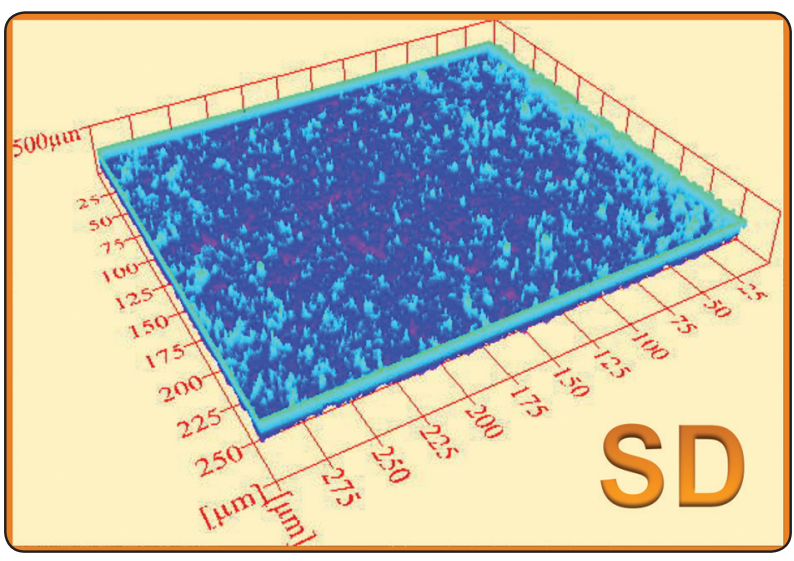

Fig. (4) Topographical assessment of Sandblasted zirconia trated surface showed increase in surface irrigularities and roughness.

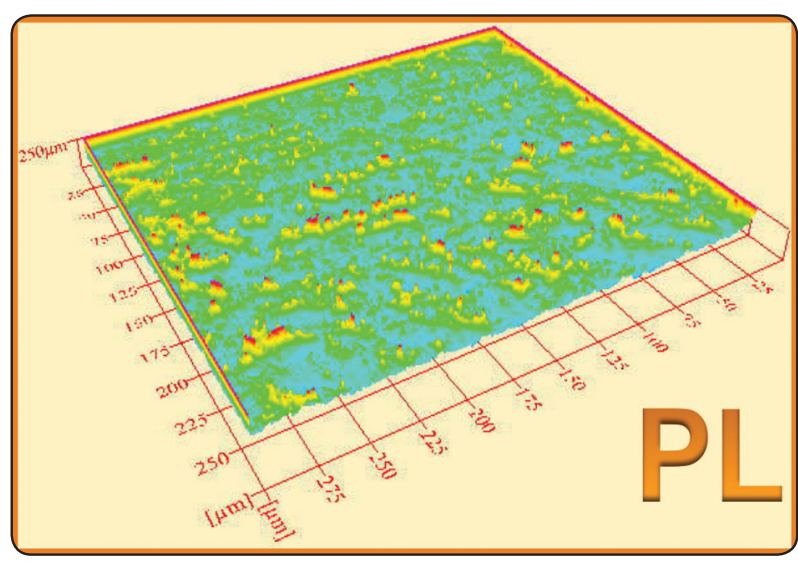

Fig. (5) Topographical assessment of air plazma zirconia trated surface showed decrease in surface irrigularities and roughness (the surface looked like it was coated with a layer) 


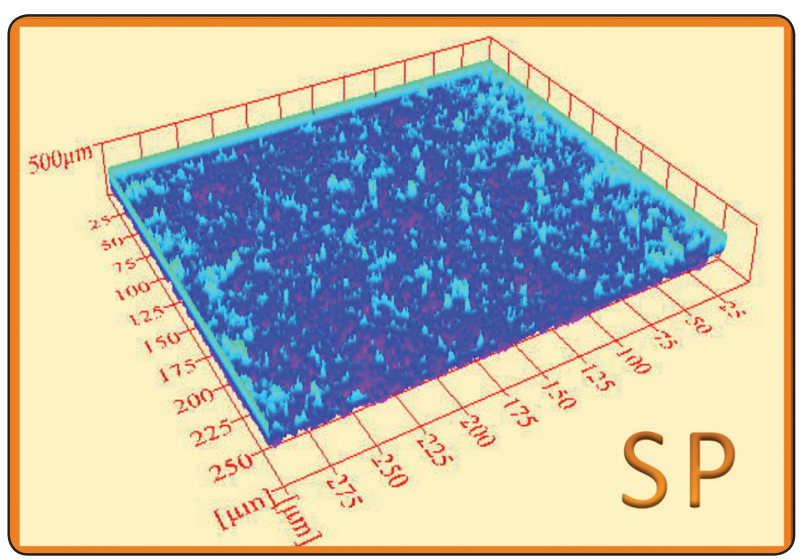

Fig. (6) Topographical assessment of sandblasted + air plazma zirconia trated surface showed increase in surface irrigularities and roughness (note that the roughness was more than the SD gp).

\section{DISCUSSION}

It's well documented that traditional adhesive strategies is not greatly effective with zirconia restorations because of the high mechanical and chemical stability. Zirconia is the restoration of choice in many clinical situations but the long term success is influenced with the proper adhesion. In 2012 Orthorp et al ${ }^{(18)}$ mentioned that after five years follow-up, zirconia single crowns reported $7 \%$ decrease in retention. Continuous attempts were carried out trying to achieve the best bond quality of cements to zirconia surface.

A systematic review in $2015^{(3)}$ concluded that sandblasting is the reference pretreatment of stronger adhesion in zirconia restoration. It was also supported by many literatures ${ }^{(19-22)}$. In last few years, plasma medicine has attracted more attention as a novel surface treatment with suggestion that the zirconia resin bond strength can be enhanced by plasma treatment ${ }^{(11 \& 23)}$. Many types of plasma treatment are available in the market with special reference to atmospheric plasma type as the simplest clinical method $^{(24)}$.

The most appropriate test for the present study was shear bond strength test (SBST) to measure the influence of the surface treatment on the changes in adhesive bond with zirconia surface ${ }^{(25)}$. This can be explained as it's well-known that tensile bond strength tests were greatly affected by the strength of the ceramic itself and not by the true adhesive bond with the resin cement ${ }^{(23)}$. Moreover, the cohesive failure within zirconia has never been reported ${ }^{(26)}$.

According to the results of the present study, the null hypothesis was accepted as a significant difference between zirconia different surface treatments were reported. SBS of SP group (sandblasting + plasma treatment) showed the highest value followed by PL group (plasma) and SD group (sandblasting). While the untreated surface (control) had the lowest SBS value (Figure 1). Furthermore, SEM findings for failure mode analysis (Figure $2 \& 3$ ) explained these results.

These were in accordance with many researches ${ }^{(5,27,28)}$. Valverde et $\mathrm{al}^{(29)}$ found a significant increase in the bond strength to zirconia surfaces when cold plasma was applied alone or in combination with alumina sandblasting. Also, Tabari et $\mathrm{al}^{(5)}$ mentioned that air plasma treatment increased the micro-shear bond strength. Ito et $\mathrm{al}^{(24)}$ referred these to increase SE (surface energy) after atmospheric plasma treatment.

Surface energy (SE) is a term describing the intermolecular forces of the material surface, depending on polar and disperse constituents of the surface $^{(30)}$. There is an opposite correlation between the SE of a surface and contact angle measurements of liquids ${ }^{(5)}$.

It's reported that different types of plasma coverage decrease the contact angle of zirconia ${ }^{(29-31)}$. With increase SE, wettability of the surface can be enhanced improving the bonding mechanism ${ }^{(29,32)}$. There are many explanation for this as increase in oxygen elements in the surface and subsequently increase polarity ${ }^{(29,33)}$. This was supported by EDX results (Table 2) as oxygen components were improved after plasma surface application. Also, plasma enhanced the development of the active 
peroxide radicals and more functional groups $(\mathrm{C}-\mathrm{O}$ and $\mathrm{C}-\mathrm{OH}$ ) on the plasma covered surfaces of inert ceramics like zirconia, which cause higher $\mathrm{SE}^{(12,16)}$. This was confirmed by XPS analysis by Noro et al study ${ }^{(34)}$.

Another mechanism for this improvement, plasma surface treatment has a cleaning influence by reducing the adsorbed hydrocarbon content, breaking of $\mathrm{C}-\mathrm{H}$ and $\mathrm{C}-\mathrm{C}$ bonds and eleminate organic contamination on the surface of zirconia ${ }^{(35)}$. The reduction of organic contaminants on zirconia treated surfaces may have played a part in enhancing the bonding of plasma treated surfaces.

These all were in accordance with many studies $^{(5,24)}$, Derand et $\mathrm{al}^{(36)}$ et al mentioned that air plasma treatment could resulted in increase the bond strength.

Contradictory to our findings, Balkenhol et $\mathrm{al}^{(23)}$ mentioned that SBS with plasma surface treatment resulted in unpredictable outcomes explaining that with integration of oxygen on surface with humidity which will inhibit the resin polymerization and supported this with spectral investigation showing $\mathrm{OH}$ content increase with $\mathrm{H}_{2} \mathrm{O}$ on zirconia surface.

Regarding sandblasting, it was considered as the most clinically applicable method for improving zirconia bond strength to adhesive resin with its simplicity. The results showed increase in bond strength than control as sandblasting could cause increase in zirconia roughness (Table1, Figure4) with more contact surface for bonding. Furthermore, it increased SE and removed organic contents of the surface which stimulating wettability. Limitations of sandblasting mainly related to creation of surface microcracks as aresult of phase transformation from tetragonal to monoclinic with subsequent effect on mechanical properties ${ }^{(3,37,38)}$.

Moreover, bonding to zirconia can be enhanced with cement containing a diphosphate monomer (Rely X Unicem cement) in conjunction with sandblasting and non-thermal plasma treatment. These enhancement in qualitative\& quantitative chemical bond with phosphate group occurs by secondary forces such as Van der Waals bond and chemical changes encouraged after plasma treatment ${ }^{(29,39)}$.

Incorporation of sandblasting and plasma application resulted in the highest SBS which can be clarified by all the above mentioned causes. Combining sandblasting with increase surface roughness (Figure 4\&6) and wettability, followed by Atmospheric non-thermal air plasma treatment which partially ionised gas in non-equilibrium providing active surface, increase SE and increase oxygen elements with more active peroxides. Both treatments can decrease organic contamination of the surface ${ }^{(29)}$.

Plasm atreatment is really a promising approach in dentistry. More researches with multiple combinations of surface treatments, thermal aging and different types of cements may be required to conclude the best method of cementing zirconia with adhesive resin.

\section{CONCLUSION}

Non thermal air plasma treatment can be used for increasing zirconia surface treatment especially when accompanied with sandblasting.

\section{Clinical significant}

Non thermal air plasma treatment alone or in combination with sandblasting can be considered as a good clinical protocol for bonding zirconia restorations.

\section{REFRANCES}

1. Piascik J, Swift EJ, Thompson J, Grego S, Stoner B. Surface modification for enhanced silanation of zirconia ceramics. Dent Mater.2009; 25(9): 1116-1121

2. M. Sasse, M. Kern. CAD/CAM single retainer zirconiaceramic resin-bonded fixed dental prostheses: clinical 
outcome after 5 years. Int. J. Comp. Dent. 2013; 16:109118.

3. E.G. Tzanakakis, I.G. Tzoutzas, P.T. Koidis, Is there a potential for durable adhesion to zirconia restorations? A systematic review. J.Prosthet. Dent. 2016;118: 9-19.

4. Lohbauer U, Zipperle M, Rischka K, Petschelt A, Müller FA. Hydroxylation of dental zirconia surfaces: characterization and bonding potential. J Biomed Mater Res B Appl Biomater. 2008;87(2):461-467.

5. Tabari K, Hosseinpour S, Mohammad-Rahimi H. The Impact of Plasma Treatment of Cercon ${ }^{\circledR}$ Zirconia Ceramics on Adhesion to Resin Composite Cements and Surface Properties. J Lasers Med Sci. 2017 Summer;8 (Supp11): S56-S61.

6. D.M. Qeblawi,M. Campillo-Funollet, C.A.Muñoz. In vitro shear bond strength of two self-adhesive resin cements to zirconia. J. Prosthet. Dent. 2015;113: 122-127.

7. Yang B, Barloi A, Kern M. Influence of air-abrasion on zirconia ceramic bonding using an adhesive composite resin. Dent Mater. 2010;26:44-50.

8. Tanaka R, Fujishima A, Shibata Y, Manabe A, Miyazaki T. Cooperation of phosphate monomer and silica modification on zirconia. J. Dent Res 2008;87:666-70.

9. G.M. de Souza, N.R. Silva, L.A. Paulillo, M.F. De Goes, E.D. Rekow, V.P. Thompson. Bond strength to high-crystalline content zirconia after different surface treatments. J. Biomed. Mater. Res. B. Appl. Biomater. 2010;93: 318-323.

10. M. Borba, P.F. Cesar, J.A. Griggs, B.A. Della, Step-stress analysis for predicting dental ceramic reliability. Dent. Mater. 2013;29: 913-918.

11. He-Ping Li , Xiao-Fei Zhang, Xiao-Ming Zhu, Miao Zheng, Shu-Fang Liu, Xuan Qi, Kai-Peng Wang, Jian Chen, Xiao-Qing Xi, Jian-Guo Tan , Kostya (Ken) Ostrikov. Translational plasma stomatology: applications of cold atmospheric plasmas in dentistry and their extension. High Volt.J. 2017;2(3):188-199.

12. Chu PK, Chen JY, Wang LP, Huang N. Plasmasurface modification of biomaterials. Mater Sci Eng R 2002;36:143-206.

13. d'Agostino R, Favia P, Oehr C, Wertheime MR. Lowtemperature plasma processing of materials: past, present, and future. Plasma Process Polym 2005;2:7-15.
14. Okubo M, Tahara M. Applications of fluoroplastics film with adhesive surface treated by atmospheric pressure nonthermal plasma hybrid process. J Adhes Soc Jpn 2010;46:116-21.

15. Heinlin J, Isbary G, Stolz W, Morfill G, Landthaler M, Shimizu T, et al. Plasma applications in medicine with a special focus on dermatology. $\mathrm{J}$ of the Europ Acad of Dermat \&Vener 2011;25:1-11.

16. Silva NR, Coelho PG, Valverde GB, Becker K, Ihrk R, Quade A. Surface characterization of Ti and Y-TZP following non-thermal plasma exposure. $\mathrm{J}$ of Biomed Mater Res Part B Appl Biomater.2011 (Oct) ;99:199-206.

17. Duan YX, Huang C, Yu QS. Cold plasma brush generated at atmospheric pressure. Review of Scientific Instruments 2007;78:27-32.

18. Ortorp, A.; Kihl, M.L.; Carlsson, G.E. A 5-year retrospective study of survival of zirconia single crowns fitted in a private clinical setting. J. Dent. 2012; 40: 527530 .

19. Blatz M, Phark J, Ozer F, Mante F, Saleh Bergler M, Sadan A. In vitro comparative bond strength of contemporary self-adhesive resin cements to zirconium oxide ceramic with and without air-particle abrasion. Clin Oral Investig 2010;14:187-92.

20. Northdurft F, Motter P, Pospiech P. Effect of surface treatment on the initial bond strength of different luting cements to zirconium oxide ceramic. Clin Oral Investig 2009;13:229-35.

21. Kitayama S, Nikaido T, Takahashi R, Zhu L, Ikeda M, Foxton RM, Sadr A, Tagami J. Effect of primer treatment on bonding of resin cements to zirconia ceramic. Dent Mater 2010;26:426-32.

22. Della Bonna A, Borba M, Benetti P, Cecchetti D. Effect of surface treatment on the bond strength of a zirconiareinforced ceramic to composite resin. Braz Oral Res 2007;21:10-5.

23. Markus Balkenhola, Frank P. Nothdurftc, Matthias Hanniga, Axel Schindlerd, Antje Lehmannd, Thomas Arnoldd, Andreas Knauberc, Stefan Rupfa,. Bonding to zirconia ceramic: The effect of cold plasma treatment and 4-META. Clin Plas Medi 2017;5: 8-13.

24. Yuki Ito, Takahisa Okawa, Takahiro Fukumoto, Akiko Tsurumi, Mitsuhiro Tatsuta, Takamasa Fujii, Junko Tanaka, Masahiro Tanaka Influence of atmospheric pressure low- 
temperature plasma treatment on the shear bond strength between zirconia and resin cement. J Prostho Res 2016;60: 289-293.

25. A. Della Bona, R. van Noort, Shear vs. tensile bond strength of resin composite bonded to ceramic, J. Dent. Res. (74) (1995) 1591-1596.

26. F.P. Nothdurft, P.J. Motter, P.R. Pospiech, Effect of surface treatment on the initial bond strength of different luting cements to zirconium oxide ceramic, Clin. Oral. Invest. (13) (2009) 229-235.

27. Singh S, Chandra R, Tripathi S, et al. The bright future of dentistry with cold plasma-review. J Dent Med Sci. 2014;13:6-13.

28. Wolfart M, Lehmann F, Wolfart S, Kern M. Durability of the resin bond strength to zirconia ceramic after using different surface conditioning methods. Dental Materials 2007;23:45-50.

29. Valverde GB, Coelho PG, Janal MN. Surface characterisation and bonding of Y-TZP following nonthermal plasma treatment. J Dent. 2013;41(1):51-59.

30. Janssen D, De Palma R, Verlaak S, Heremans P, Dehaen W. Static solvent contact angle measurements, surface free energy and wettability determination of various selfassembled monolayers on silicon dioxide. Thin Solid Films. 2006;515(4):1433-1438.

31. Amaral R, Ozcan M, Bottino MA, Valandro LF Microtensile bond strength of a resin cement to glass infiltrated zirconia reinforced ceramic: the effect of surface conditioning. Dentl Mater 2006;22:283-90.

32. Marcos Daniel Septímio Lanzaa, Flavia Juliani Souza
Rodrigues Lanzab, Adriana Pigozzo Mansoc, Jukka Pekka Matinlinnad, Ricardo Marins Carvalho. Innovative surface treatments for improved ceramic bonding: Lithium disilicate glass ceramic. Inter J Adhes\& Adhesiv 2018;82: 60-66.

33. Fridman A. Plasma Chemistry. New York: Cambridge University Press; 2008.

34. Noro A, Kaneko M, Murata I, Yoshinari M. Influence of surface topography and surface physicochemistry on wettability of zirconia (tetragonal zirconia polycrystal). J Biomed Mater Res B Appl Biomater. 2013;101(2):355363.

35. Wu C-C, Wei C-K, Ho C-C, Ding S-J. Enhanced hydrophilicity and biocompatibility of dental zirconia ceramics by oxygen plasma treatment. Mater. 2015; 8(2):684-699.

36. Derand T, Molin M, Kvam K. Bond strength of composite luting cement to zirconia ceramic surfaces. Dent Mater. 2005;21(12):1158-1162.

37. Blatz MB, Chiche G, Holst S, Sadan A. Influence of surface treatment and simulated aging on bond strengths of luting agents to zirconia. Quintes Inter 2007;38:745-53.

38. Esteves-Oliveira, M.; Jansen, P.;Wehner, M.; Dohrn, A.; Bello-Silva, M.S.; Eduardo, C.P.; Meyer-Lueckel, H. Surface characterization and short-term adhesion to zirconia after ultra-short pulsed laser irradiation. J. Adhes. Dent. 2016;18: 483-492.

39. Luthy H,Loeffel O,Hammerle CH. Effect of thermocycling on bond strength of luting cements to zirconia ceramic. Dent Mater 2006;22:195-200. 